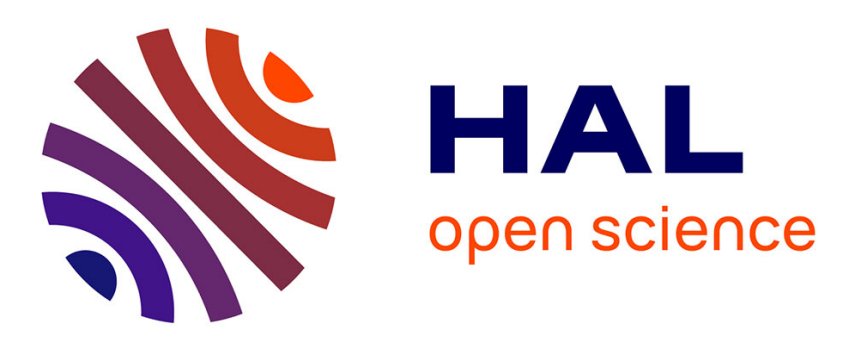

\title{
A constitutive model for rubber bearings subjected to combined compression and shear
}

R. Bouaziz, C Ovalle, L. Laiarinandrasana

\section{To cite this version:}

R. Bouaziz, C Ovalle, L. Laiarinandrasana. A constitutive model for rubber bearings subjected to combined compression and shear. Constitutive Models for Rubber XI, 1, CRC Press, pp.255-260, 2019, 10.1201/9780429324710-45. hal-02520247

\section{HAL Id: hal-02520247 \\ https://hal.science/hal-02520247}

Submitted on 26 Mar 2020

HAL is a multi-disciplinary open access archive for the deposit and dissemination of scientific research documents, whether they are published or not. The documents may come from teaching and research institutions in France or abroad, or from public or private research centers.
L'archive ouverte pluridisciplinaire HAL, est destinée au dépôt et à la diffusion de documents scientifiques de niveau recherche, publiés ou non, émanant des établissements d'enseignement et de recherche français ou étrangers, des laboratoires publics ou privés. 


\title{
A constitutive model for rubber bearings subjected to combined compression and shear
}

\author{
R. Bouaziz, C. Ovalle and L. Laiarinandrasana \\ PSL Research University \\ Mines ParisTech, Centre des Matriaux, CNRS 7633 BP 87, F-91003 Evry Cedex, France
}

\begin{abstract}
This paper focuses on the finite strain thermo-mechanical modelling of the dynamical behaviour of filled rubbers under combined compression and shear using a multiphysics coupling approach. The mechanical behaviour of such materials depends on the network evolution due to thermo-mechanical loadings and the external environmental conditions (oxidation, ageing ...). An experimental campaign including tensile, compression/shear and compression/torsion tests allows the study of the impact of thermal ageing on the material properties, namely the bulk modulus and the shear modulus. This experimental investigation is dedicated to characterize the relationship between the hydrostatic pressure versus the volume change and the isochoric visco-hyperelastic behaviour as a function of ageing.
\end{abstract}

\section{INTRODUCTION}

Laminated rubber bearings are commonly used for the seismic isolation of civil engineering structures. During earthquake, the rubber material is supposed to undergo static compressive loading combined with cyclic shear. In terms of constitutive modelling, rubber is generally assumed to be quasi-incompressible. Nevertheless, in the laminated geometry of bearings, exhibiting a high shape factor (Dorfmann, Fuller, \& Ogden 2002), modelling the relationship between the hydrostatic pressure versus the volume change is of prime importance. In this contribution, an attempt is made to investigate a relevant constitutive model, using a multiphysics coupling approach, such as the one developed in (Lejeunes \& Eyheramendy 2018), that allows the combined shear and dilational responses to be handled. To this end, an original experimental setup is used to obtain a reliable database, useful for the finite element modelling.

On the other hand, during long term service, the shear and bulk moduli of the rubber can evolve due to environmental influences such as temperature, humidity, oxygen... The evolution of these mechanical properties can be related to the modification of the rubber network due to the chemical-mechanical interaction (Steinke et al. 2011). In a second part of the study, ageing on rubber samples will be replicated by means of an experimental set-up based on degradation of the material by oxidative ageing. The results will be used to enhance the proposed model by offering a closer look at the ageing effects on the rubber mechanical properties. This may help to improve the residual life-time prediction for related applications.

\section{CONSTITUTIVE MODELLING}

To take the thermal ageing into account within the thermo-mechanical constitutive model, a similar approach as (NGuyen, Lejeunes, Eyheramendy, \& Boukamel 2016) is adopted. A local chemo-physical evolution describing ageing kinetics by means of a chemical internal variable designated by $\xi$ is introduced. $\xi(\mathbf{x}, t)$ is a local chemical conversion variable $(\xi \in[0,1]$ and $\xi(\mathbf{x}, 0)=0)$, in which $\mathbf{x}$ is the current position of a material point. This variable describes the network evolution (increase of the apparent sulphur crosslink density, oxidation, chain breakage ...) during thermal ageing. As in (Lejeunes, Eyheramendy, Boukamel, Delattre, Méo, \& Ahose 2018) the evolution of the apparent crosslink density depends on both thermal and mechanical states.

\section{$2.1 \quad$ Kinematics}

The proposed constitutive model is based on the thermodynamics of irreversible processes and on the local state assumption. Viscoelastic overstress is introduced through an intermediate configuration defined from the splitting of the deformation gradient $\mathbf{F}(\mathbf{X}, t)$, where $\mathbf{X}$ is the position of a material point in the reference configuration. The deformation gradient $\mathbf{F}$ is split into a volumetric and an isochoric part. The iso- 
choric part is decomposed multiplicatively into purely isochoric elastic parts $\overline{\mathbf{F}}_{\mathbf{e}}^{i}(\mathbf{X}, t)$ and isochoric viscous ones $\overline{\mathbf{F}}_{\mathbf{v}}^{i}(\mathbf{X}, t)$, where $i$ stands for the $i^{t h}$ decomposition:

$$
\left\{\begin{array}{l}
\mathbf{F}=\mathbf{F}^{v o l} \overline{\mathbf{F}} \\
\mathbf{F}^{v o l}=J^{1 / 3} \mathbf{1} \\
\overline{\mathbf{F}}=J^{-1 / 3} \mathbf{F}=\overline{\mathbf{F}}_{\mathbf{e}^{i}} \overline{\mathbf{F}}_{\mathbf{v}}^{i}
\end{array}\right.
$$

where:

- $\mathbf{F}^{\text {vol }}$ and $\overline{\mathbf{F}}$ are respectively the volumetric and the isochoric parts of the deformation gradient F.

- $\mathbf{1}$ is the second order unit tensor.

- $J=\operatorname{det}(\mathbf{F})$ is the jacobian of $\mathbf{F}$ that corresponds to the total volume variation.

From physical measurements (mass and dimension) of samples before and after ageing, no ageingdependent variation of mass or volume was observed. Therefore, we assume that volume variation $J$ can only be attributed to thermal dilatation or mechanical compressibility. We adopt the following splitting of the volume variation $J=J_{\Theta} J_{m}$, with

$$
\begin{aligned}
& J_{\Theta}=1+\alpha_{\Theta}\left(\Theta-\Theta_{0}\right) \\
& J_{m}=\frac{J}{J_{\Theta}}
\end{aligned}
$$

where $\alpha_{\Theta}$ is the thermal expansion coefficient, $\Theta_{0}$ is the reference temperature and $\Theta$ is the current temperature. The previous linear expansion relation is motivated by experimental evidences that we have obtained from dilatometry experiments.

\subsection{Thermodynamics}

In the following, the hybrid free energy concept (Lion et al. 2014, Lejeunes and Eyheramendy 2018) is adopted. The main idea is to formulate the free energy from standard state and internal variables. The volume dependency is replaced by a pressure-like variable designated by $q$. This formalism can be viewed as a generalisation of partial Legendre transformation of the free energy. lows:

The hybrid free specific energy is assumed as fol-

$$
\begin{aligned}
\varphi\left(\overline{\mathbf{B}}, \overline{\mathbf{B}}_{\mathbf{e}}^{i}, q, \xi, \Theta\right) & =\varphi_{i s o}\left(\overline{\mathbf{B}}, \overline{\mathbf{B}}_{\mathbf{e}}^{i}, \xi, \Theta\right) \\
& +\varphi_{v o l}(q, \Theta)+\varphi_{t h}(\Theta) \\
& +\varphi_{\text {chem }}(\xi, \Theta)
\end{aligned}
$$

where $\overline{\mathbf{B}}=\overline{\mathbf{F}} \cdot \overline{\mathbf{F}}^{\mathrm{T}}$ and $\overline{\mathbf{B}}_{\mathbf{e}}^{i}=\overline{\mathbf{F}}_{\mathbf{e}}^{i} \cdot \overline{\mathbf{F}}_{\mathrm{e}}^{i^{\mathrm{T}}}$ are respectively the isochoric left Cauchy Green tensor and the $i^{t h}$ elastic isochoric left Cauchy Green tensor.
The additive splitting of (4) is motivated by the fact that it can exist stress-free purely thermal variations for which no chemical reaction occur (contribution of $\left.\varphi_{t h}\right)$. Stress-free thermochemical evolutions can also be observed (contribution $\varphi_{\text {chem }}$ ) and finally thermomechanical states can also exist without any chemical reaction. Furthermore, due to the huge difference of stiffness for both hydrostatic or isochoric states, Flory (1961) isochoric/volumetric splitting is followed and adopted for the remaining part of the hybrid energy (contributions of $\varphi_{i s o}$ and $\varphi_{v o l}$ ). $\varphi_{t h}$ is assumed to depend only on $\Theta$. This is the same for the volumetric contribution, therefore $\varphi_{v o l}$ depends only on the pressure-like variable $q$ and temperature.

The hybrid energy approach requires to define a supplementary potential $\beta$, which allows to relate $q, J$ and $\Theta$ with a constitutive law, or a supplementary flow rule, if a dissipative mechanism in volume is considered, cf. (Lejeunes and Eyheramendy 2018) for more details. The hybrid free energy $\varphi$ can be related to the Helmholtz free energy $\psi$ through the relation:

$$
\begin{aligned}
\psi\left(\overline{\mathbf{B}}, \overline{\mathbf{B}}_{\mathbf{e}}^{i}, J, \xi, \Theta\right) & =\varphi\left(\overline{\mathbf{B}}, \overline{\mathbf{B}}_{\mathbf{e}}^{i}, q, \xi, \Theta\right) \\
& -\beta(J, \Theta, q)
\end{aligned}
$$

The combination of the first and the second law of thermodynamics, in the Clausius-Duhem form, leads to the following inequality:

$$
\begin{gathered}
\phi=\rho\left(\frac{\boldsymbol{\sigma}}{\rho}-2\left(\overline{\mathbf{B}} \frac{\partial \varphi}{\partial \overline{\mathbf{B}}}+\sum_{i=1}^{N} \overline{\mathbf{B}}_{\mathbf{e}}^{i} \frac{\partial \varphi}{\partial \overline{\mathbf{B}}_{\mathbf{e}}^{i}}\right)^{\mathrm{D}}+J \frac{\partial \beta}{\partial J} \mathbf{1}\right): \mathbf{D} \\
-\quad \rho\left(s+\frac{\partial \varphi}{\partial \Theta}-\frac{\partial \beta}{\partial \Theta}\right) \dot{\Theta}+2 \rho \sum_{i=1}^{N}\left(\overline{\mathbf{B}}_{\mathbf{e}}^{i} \frac{\partial \varphi}{\partial \overline{\mathbf{B}}_{\mathbf{e}}^{i}}\right): \overline{\mathbf{D}}_{\mathbf{v}}^{\mathbf{o} i} \\
-\quad \rho\left(\frac{\partial \varphi}{\partial q}-\frac{\partial \beta}{\partial q}\right) \dot{q}-\rho \frac{\partial \varphi}{\partial \xi} \dot{\xi}-\frac{\operatorname{grad}_{x} \Theta}{\Theta} \mathbf{q}_{\Theta} \geq 0 \\
\forall \mathbf{D}, \dot{\Theta}, \overline{\mathbf{D}}_{\mathbf{v}}^{\mathbf{o} i}, \dot{q}, \mathbf{q}_{\Theta} \dot{\xi}
\end{gathered}
$$

where $\phi$ is the total dissipation, $\boldsymbol{\sigma}$ is the Cauchy stress, $\mathbf{D}=1 / 2\left(\mathbf{L}+\mathbf{L}^{\mathrm{T}}\right)$ is the eulerian strain rate with $\mathbf{L}=\dot{\mathbf{F}} \mathbf{F}^{-1}, \rho$ is the current density, $s$ is the specific entropy, $\mathbf{q}_{\Theta}$ is the Eulerian heat flux, $\overline{\mathbf{D}}_{\mathbf{v}}^{\mathbf{o} i}$ is the $i^{t h}$ objective eulerian strain rate, defined by:

$$
\overline{\mathbf{D}}_{\mathbf{v}}^{\mathbf{o}^{i}}=\overline{\mathbf{R}}_{\mathbf{e}}^{i} \overline{\mathbf{D}}_{\mathbf{v}}^{i} \overline{\mathbf{R}}_{\mathbf{e}}^{i^{\mathrm{T}}}
$$

where $\overline{\mathbf{R}}_{\mathrm{e}}^{i}$ comes from the polar decomposition of $\overline{\mathbf{F}}_{\mathbf{e}}^{i}=\overline{\mathbf{V}}_{\mathbf{e}}^{i} \overline{\mathbf{R}}_{\mathbf{e}}^{i}$.

Assuming that the dissipation is only due to thermal diffusion, mechanical viscosity including Payne effect and chemical evolution, the following constitutive equations can be obtained from (6): 
$\boldsymbol{\sigma}=\overbrace{2 \rho\left(\overline{\mathbf{B}} \frac{\partial \varphi_{\text {iso }}}{\partial \overline{\mathbf{B}})^{\mathrm{D}}+\frac{q}{J_{\Theta}} \mathbf{1}}\right.}^{\boldsymbol{\sigma}_{e q}}+\overbrace{\sum_{i=1}}^{N} \overbrace{2 \rho\left(\overline{\mathbf{B}}_{\mathbf{e}}^{i} \frac{\partial \varphi_{\text {iso }}}{\partial \overline{\mathbf{B}}_{\mathbf{e}}^{i}}\right)^{\mathrm{D}}}^{\boldsymbol{\sigma}_{v}^{i}}$

$s=-\frac{\partial \varphi}{\partial \Theta}+\frac{\partial \beta}{\partial \Theta}$

$\frac{\partial \varphi_{v o l}}{\partial q}=\frac{\partial \beta}{\partial q}$

where $\boldsymbol{\sigma}_{e q}$ is the time-independent equilibrium stress at which the total stress $\boldsymbol{\sigma}$ is totally relaxed, i.e. $\boldsymbol{\sigma}_{e q}=$ $\boldsymbol{\sigma}(t \rightarrow \infty), \boldsymbol{\sigma}_{v}^{i}$ is the $i^{t h}$ viscous (non-equilibrium) stress, i.e. $\boldsymbol{\sigma}_{v}^{i}(t \rightarrow \infty)=0$. The pressure-like variable $q$ is defined by (10) and from (8) the hydrostatic pressure is defined $p=q / J_{\Theta}$.

It is considered that internal variables evolve independently from each other, e.g. (Germain, Nguyen, \& Suquet 1983); then, the positivity of each dissipation term is independently required. From (6), the following inequalities are returned:

$$
\begin{aligned}
\phi_{v i s}^{i} & =2 \rho\left(\overline{\mathbf{B}}_{\mathbf{e}}^{i} \frac{\partial \varphi_{i s o}}{\partial \overline{\mathbf{B}}_{\mathbf{e}}^{i}}\right): \overline{\mathbf{D}}_{\mathbf{v}}^{\mathbf{o}^{i}} \\
& =\boldsymbol{\sigma}_{v}^{i}: \overline{\mathbf{D}}_{\mathbf{v}}^{\mathbf{o}^{i}} \geq 0 \quad \forall \overline{\mathbf{D}}_{\mathbf{v}}^{\mathbf{o}^{i}} \quad i=1 . . N \\
\phi_{\xi}= & -\rho \frac{\partial \varphi}{\partial \xi} \dot{\xi}=A_{\xi} \dot{\xi} \geq 0 \quad \forall \dot{\xi} \\
\phi_{\Theta} & =\left(-\frac{\operatorname{grad}_{\boldsymbol{x}} \Theta}{\Theta}\right) \mathbf{q}_{\Theta}=A_{\Theta} \mathbf{q}_{\Theta} \geq 0 \quad \forall \mathbf{q}_{\Theta}
\end{aligned}
$$

where $\boldsymbol{\sigma}_{v}^{i}, A_{\xi}, A_{\Theta}$ are thermodynamical forces associated with the thermodynamical fluxes $\overline{\mathbf{D}}_{\mathbf{v}}^{\mathbf{o}^{i}}, \dot{\xi}, \mathbf{q}_{\Theta}$. For the thermal dissipation term, i.e. 13, an isotropic Fourier conduction law such as is assumed:

$\mathbf{q}_{\Theta}=-k_{\Theta} \operatorname{grad}_{\boldsymbol{x}} \Theta$

The conductivity coefficient $k_{\Theta}$ is assumed to be constant and not affected by ageing. There are not experimental evidences supporting this assumption; however, results can be used as an initial approximation.

\subsection{Mechanical behaviour}

An isotropic behaviour is considered with the following potentials:

$$
\begin{aligned}
\rho_{0} \varphi_{\text {iso }} & =C_{10}\left(I_{1}(\overline{\mathbf{B}})-3\right)+C_{01}\left(I_{2}(\overline{\mathbf{B}})-3\right) \\
& +\sum_{i=1}^{N} \frac{\mu_{i}}{2}\left(I_{1}\left(\overline{\mathbf{B}}_{\mathbf{e}}^{i}\right)-3\right)
\end{aligned}
$$

$\rho_{0} \varphi_{v o l}=-\frac{1}{2} k\left(1+\frac{q}{k}\right)^{2}+q+k$

$\rho_{0} \beta=q\left(1-J_{m}\right)$

where $C_{10}, C_{20}$ are the Mooney-Rivlin coefficients that are assumed to be dependent on $\xi$ and $\Theta, \mu_{i}$ is the $i^{\text {th }}$ shear modulus that depends on $\xi$ and $\Theta$. The bulk modulus $k$ is assumed to be independent on these variables. $I_{1}$ and $I_{2}$ are respectively the first and the second invariant of the strain tensor and $\rho_{0}=J \rho$ is the initial mass density.

\subsubsection{Equilibrium contribution}

From (8) the equilibrium stress is written as:

$$
\begin{aligned}
\boldsymbol{\sigma}_{e q} & =2 J^{-1} C_{10} \overline{\mathbf{B}}^{\mathrm{D}} \\
& +2 J^{-1} C_{01}\left(I_{1}(\overline{\mathbf{B}}) \overline{\mathbf{B}}-\overline{\mathbf{B}}^{2}\right)^{\mathrm{D}}+\frac{q}{J_{\Theta}} \mathbf{1}
\end{aligned}
$$

\subsubsection{Visco-elastic contribution}

From (8) the $i^{\text {th }}$ viscous stress is written as:

$$
\boldsymbol{\sigma}_{v}^{i}=J^{-1} \mu_{i} \overline{\mathbf{B}}_{\mathbf{e}}^{i \quad \mathrm{D}} \quad i=1 . . N
$$

The following Maxwell-like flow rule for viscosity is considered :

$$
\overline{\mathbf{D}}_{\mathbf{v}}^{\mathbf{o}^{i}}=\frac{1}{\eta_{i}} \boldsymbol{\sigma}_{v}^{i} \quad i=1 . . N
$$

where $\eta_{i}$ is the $i^{\text {th }}$ viscosity parameter.

The positivity of the viscous dissipation terms $\phi_{v i s}^{i}$ can be easily shown by inserting (20) in (11). From the time differentiation of $\overline{\mathbf{B}}_{\mathbf{e}}^{i}$ and the multiplicative splitting of the deformation gradient, the Maxwell flow rule can be also reformulated by the following relation:

$\dot{\overline{\mathbf{B}}}_{\mathbf{e}}{ }^{i}=\mathbf{L} \overline{\mathbf{B}}_{\mathbf{e}}^{i}+\overline{\mathbf{B}}_{\mathbf{e}}^{i} \mathbf{L}^{\mathrm{T}}-2 \overline{\mathbf{V}}_{\mathbf{e}}^{i} \overline{\mathbf{D}}_{\mathbf{v}}^{\mathbf{o}^{i}} \overline{\mathbf{V}}_{\mathbf{e}}^{i}$

Combining (20) and (21), we can obtain:

$$
\left\{\begin{array}{l}
\bullet \dot{\overline{\mathbf{B}}}_{\mathbf{e}}{ }^{i}=\mathbf{L} \overline{\mathbf{B}}_{\mathbf{e}}^{i}+\overline{\mathbf{B}}_{\mathbf{e}}^{i} \mathbf{L}^{\mathrm{T}} \\
\quad-\frac{2}{3}(\mathbf{1}: \mathbf{L}) \overline{\mathbf{B}}_{\mathbf{e}}^{i}-\frac{1}{J \tau_{i}} \overline{\mathbf{B}}_{\mathbf{e}}^{i} \overline{\mathbf{B}}_{\mathbf{e}}^{i \quad \mathrm{D}} \quad i=1 . . N \\
\bullet \overline{\mathbf{B}}_{\mathbf{e}}^{i}(t=0)=\mathbf{1}
\end{array}\right.
$$

where $\tau_{i}=\eta_{i} / 2 \mu_{i}$ is a characteristic time of viscosity linked to the $i^{t h}$ viscous stress. 
3 APPLICATION TO A

COMPRESSION-TORSION TEST

\subsection{Analytical modelling}

An homogeneous cylinder (Figure 1) with $L$ as initial length and $A$ as initial radius is considered. In the initial configuration $C_{0}$, a material point $\mathrm{M}$ of the undeformed cylinder is identified by $\left(R, \theta_{0}, Z\right)$ in the initial cylindrical coordinate system $\left(e_{R}, e_{\theta_{0}}\right.$, $\left.e_{Z}\right)$. The transformation consists of an axial displacement in the $e_{Z}$ direction and a torsion angle around the same vector $\left(e_{Z}\right)$ applied on the upper surface $S_{u}$. The lower suface $S_{l}$ is maintained fixed and the lateral surface $S_{\text {lat }}$ is free. The length and radius of the deformed cylinder are noted respectively by $l$ and $a$. By this transformation, the point $M$ becomes $M^{\prime}$, identified by $(r, \theta, z)$ in the deformed cylindrical coordinate system $\left(e_{r}, e_{\theta}, e_{z}\right)$.

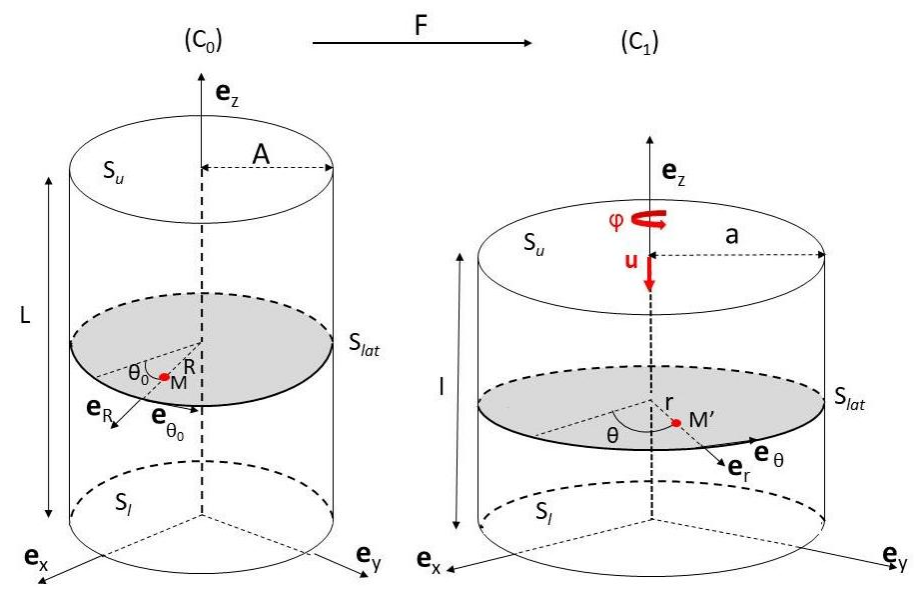

Figure 1: Kinematics of a cylinder subjected to compression/torsion

The displacement of $M$ to $M^{\prime}$ is described by:

$r=\frac{R}{\sqrt{\lambda}}, \quad \theta=\theta_{0}+\tau Z$

where $\lambda$ is the relative elongation and $\tau$ is the angle per unit of initial length:

$\lambda=\frac{l}{L}, \quad \tau=\frac{\phi}{L}$

Considering the case of combined oedometric compression and torsion, the deformation gradient can be written as:

$\mathbf{F}=\left[\begin{array}{ccc}1 & 0 & 0 \\ 0 & 1 & \tau r \\ 0 & 0 & \lambda\end{array}\right]$

and the Cauchy Green tensor is given by:

$\mathbf{B}=\left[\begin{array}{ccc}1 & 0 & 0 \\ 0 & 1+\tau^{2} r^{2} & \lambda \tau r \\ 0 & \lambda \tau r & \lambda^{2}\end{array}\right]$
The viscoelastic Cauchy Green tensor takes the following form:

$$
\overline{\mathbf{B}}_{\mathbf{e}}^{i}=\left[\begin{array}{ccc}
1 & 0 & 0 \\
0 & \bar{B}_{e_{22}^{i}}^{i} & \bar{B}_{e_{23}}^{i} \\
0 & \bar{B}_{e_{23}}^{i} & \bar{B}_{e_{33}}^{i}
\end{array}\right]
$$

Inserting (27) in (22), a system of differential equations with three unknowns is obtained.

Let us consider a disc with a radius $A=12.5 \mathrm{~mm}$ and an initial length $L=2 \mathrm{~mm}$. The initial shape factor is given by $S_{f}=A / L=6.25$. This disc is submitted to compression by imposing $\lambda=0.9$ and torsion by imposing a rotation angle $\phi=10 \pi / 180$ on the upper surface.

\subsection{Discussion}

In order to study the contribution of shear stress and the hydrostatic pressure as a function of ageing, an increase of both the bulk modulus $K$ and the shear modulus $G$ with ageing is assumed.

An attempt was made first to plot the evolution of the shear stress and the hydrostatic pressure (absolute value) as a function of the normalized radius for different arbitrary values of $G$ and $K$, obtained from the analytical model. However, in case of compression loading on a disc having a shape factor higher than 5 , the hydrostatic pressure curve should not be constant near the edge of the disc due to the well-known barrelling effect (Dorfmann, Fuller, \& Ogden 2002). Assuming that the absolute value of the hydrostatic pressure decreases between $0.8 a$ and $a$, Figure 2 illustrates the expected curves of shear stress and hydrostatic pressure as a function of the normalized radius.

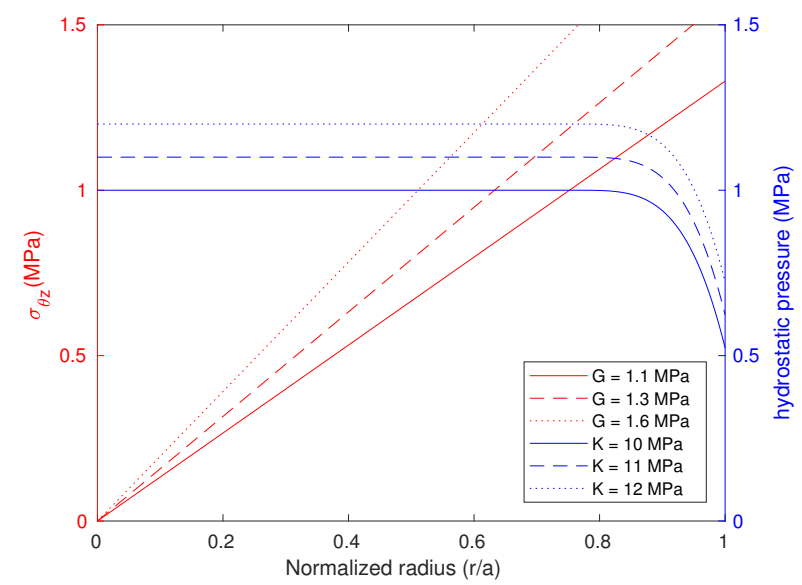

Figure 2: Evolution of the shear stress and the hydrostatic pressure as a function of the normalized radius: (*expected appearance of hydrostatic curves)

On the other hand, the stress triaxiality ratio is defined as follows:

$\chi=\frac{p}{\sigma_{\theta z}}$ 
where $p$ is the hydrostatic pressure and $\sigma_{\theta z}$ is the shear stress.

Figure 3 shows the evolution of the normalized radius at a fixed bulk modulus $K=10 \mathrm{MPa}$ and a stress triaxiality ratio $\chi=1$ as a function of the shear modulus $G$. The normalized radius non-linear decreases with an increase of $G$.

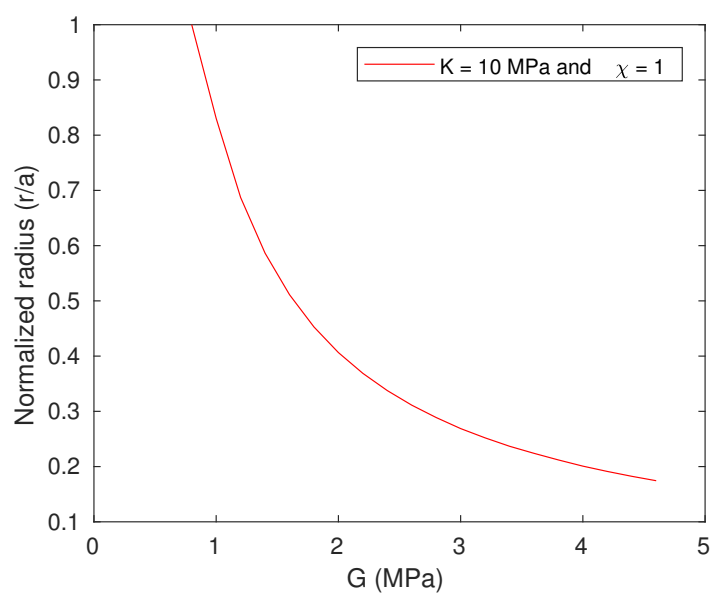

Figure 3: Evolution of the normalized radius for $K=$ cte and $\chi=1$ as a function of $G$

Figure 4 shows the evolution of the stress triaxiality ratio $\chi$ at a fixed bulk modulus $K=10 \mathrm{MPa}$ for a radius $r=0.8 a$ as a function of the shear modulus $G$. The stress triaxiality ratio increases linearly with an increase of $G$.

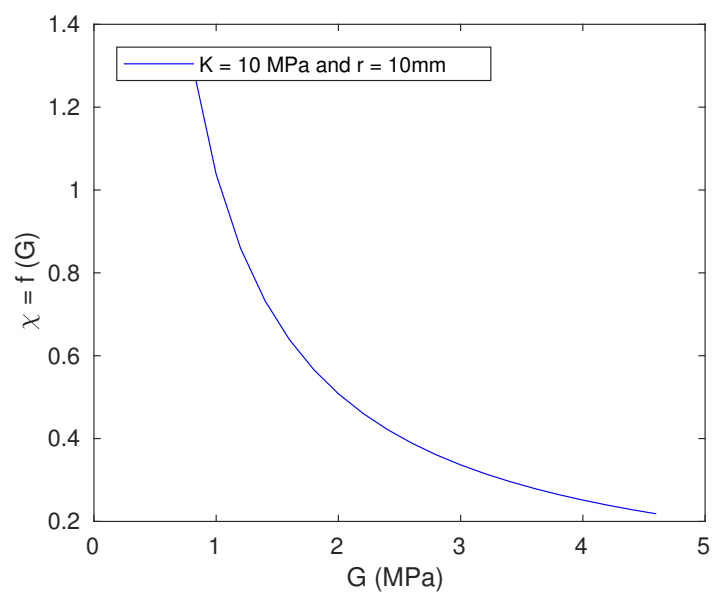

Figure 4: Evolution of $\chi$ for $K=c t e$ and $r=0.8 a$ as a function of $G$

Figure 5 shows the evolution of the normalized radius $r$ at a fixed shear modulus $G=1.1 \mathrm{MPa}$ and a stress triaxiality ratio $\chi=1$ as a function of the bulk modulus $K$. The normalized radius increases linearly with an increase of $K$.

Figure 6 shows the evolution of the ratio $\chi$ at a fixed shear modulus $G=1.1 \mathrm{MPa}$ and a radius $r=0.8 a$ as a function of the bulk modulus $K$. The stress triaxiality ratio non-linear decreases with an increase of $K$.

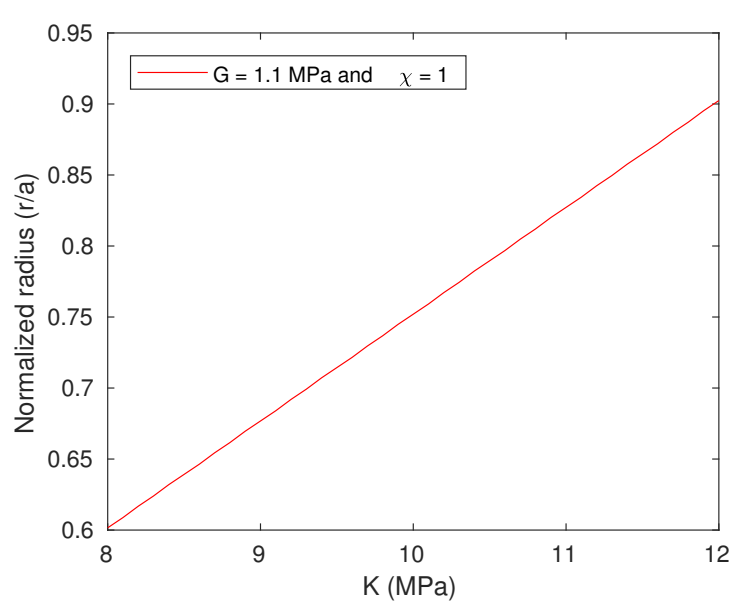

Figure 5: Evolution of the normalized radius for $G=$ cte and $\chi=1$ as a function of $K$

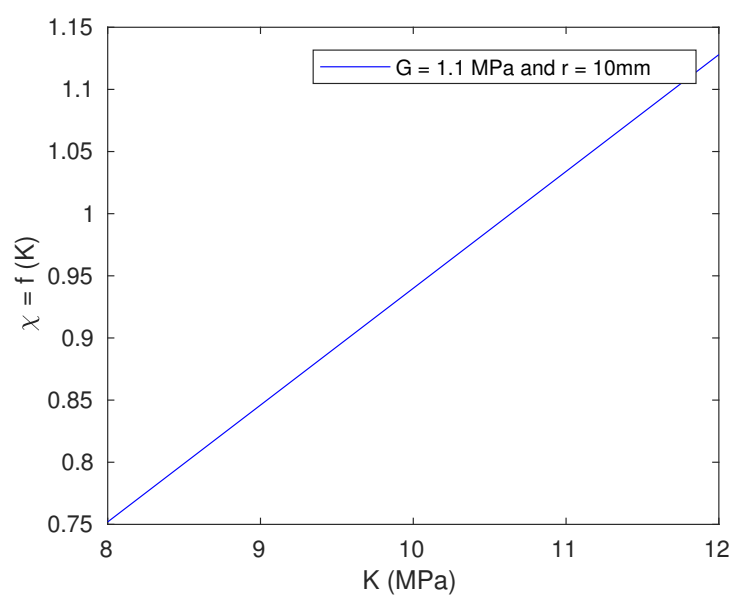

Figure 6: Evolution of $\chi$ for $G=c t e$ and $r=0.8 a$ as a function of $K$

\section{CONCLUSIONS}

In this paper, we presented a constitutive model based on a multiphysics coupling approach for compressible filled rubbers. This model takes into account the network mechanisms that are responsible of the evolution of the mechanical properties. The present work showed the trend of the stress triaxiality ratio when the bulk and shear moduli are supposed to increase due to ageing. Finally, in a future work, the model will be implemented into a finite element code and verified with experimental results. Moreover, the time-dependent deformation (viscohyperelastic behaviour) will be studied and included on both volumetric and isochoric responses.(Bouaziz, Ahose, Lejeunes, Eyheramendy, \& Sosson 2019)

\section{REFERENCES}

Bouaziz, R., K. Ahose, S. Lejeunes, D. Eyheramendy, \& F. Sosson (2019). Characterization and modeling of filled rubber submitted to thermal aging. International Journal of Solids and Structures 169, $122-140$.

Dorfmann, A., K. Fuller, \& R. Ogden (2002). Shear, compressive and dilatational response of rubberlike solids subject to cavitation damage. International Journal of Solids and Structures 39, 1845-1861. 
Flory, R. J. (1961). Thermodynamic relations for highly elastic materials. Transactions of the Faraday Society 57, 829-838.

Germain, P., Q. Nguyen, \& P. Suquet (1983). Continuum thermodynamics. Journal of Applied Mechanics 50, 1010-1020.

Lejeunes, S. \& D. Eyheramendy (2018, May). Hybrid free energy approach for nearly incompressible behaviors at finite strain. Continuum Mechanics and Thermodynamics.

Lejeunes, S., D. Eyheramendy, A. Boukamel, A. Delattre, S. Méo, \& K. D. Ahose (2018, Feb). A constitutive multiphysics modeling for nearly incompressible dissipative materials: application to thermo-chemo-mechanical aging of rubbers. Mechanics of Time-Dependent Materials 22(1), 51-66.

Lion, A., B. Dippel, \& C. Liebl (2014). Thermomechanical material modelling based on a hybrid free energy density depending on pressure, isochoric deformation and temperature. International Journal of Solids and Structures 51(34), 729 739.

NGuyen, T., S. Lejeunes, D. Eyheramendy, \& A. Boukamel (2016). A thermodynamical framework for the thermochemo-mechanical couplings in soft materials at finite strain. Mechanics of Materials 95, $158-171$.

Steinke, L., J. Spreckels, M. Flamm, \& M. Celina (2011). Model for heterogeneous aging of rubber products. Plastics, Rubber and Composites 40(4), 175-179. 